\title{
Associations between the probabilities of frequency-specific hearing loss and unaided APHAB scores
}

\author{
J. Löhler ${ }^{1,2} \cdot$ B. Wollenberg ${ }^{2} \cdot$ P. Schlattmann ${ }^{3} \cdot$ N. Hoang ${ }^{3} \cdot$ R. Schönweiler ${ }^{4}$
}

Received: 4 October 2016/ Accepted: 9 November 2016/Published online: 17 November 2016

(C) The Author(s) 2016. This article is published with open access at Springerlink.com

\begin{abstract}
The Abbreviated Profile of Hearing Aid Benefit (APHAB) questionnaire reports subjective hearing impairments in four typical conditions. We investigated the association between the frequency-specific probability of hearing loss and scores from the unaided APHAB $\left(\mathrm{APHAB}_{\mathrm{u}}\right)$ to determine whether the $\mathrm{APHAB}_{\mathrm{u}}$ could be useful in primary diagnoses of hearing loss, in addition to pure tone and speech audiometry. This retrospective study included database records from 6558 patients (average age 69.0 years). We employed a multivariate generalised linear mixed model to analyse the probabilities of hearing losses (severity range $20-75 \mathrm{~dB}$, evaluated in 5-dB steps), measured at different frequencies $(0.5,1.0,2.0,4.0$, and $8.0 \mathrm{kHz}$ ), for nearly all combinations of $\mathrm{APHAB}_{\mathrm{u}}$ subscale scores (subscale scores from 20 to $80 \%$, evaluated in steps of $5 \%$ ). We calculated the probability of hearing loss for 28,561 different combinations of $\mathrm{APHAB}_{\mathrm{u}}$ subscale scores (results available online). In general, the probability of
\end{abstract}

Electronic supplementary material The online version of this article (doi:10.1007/s00405-016-4385-7) contains supplementary material, which is available to authorized users.

\section{J. Löhler}

loehler@hno-aertze.de

1 Scientific Institute for Applied ENT-Research of the German Professional Association of ENT-Surgeons, Maienbeeck 1, 24576 Bad Bramstedt, Germany

2 Department of ENT-Surgery, Universital Hospital Schleswig-Holstein, Campus Luebeck, Lübeck, Germany

3 Institute for Medical Statistics, Informatics and Documentations, Universital Hospital Jena, Campus Luebeck, Jena, Germany

4 Section of Phoniatrics and Pedaudiology in the Department of ENT-Surgery, Universital Hospital of Schleswig-Holstein, Campus Luebeck, Lübeck, Germany hearing loss was positively associated with the combined $\mathrm{APHAB}_{\mathrm{u}}$ score (i.e. increasing probability with increasing scores). However, this association was negative at one frequency $(8 \mathrm{kHz})$. The highest probabilities were for a hearing loss of $45 \mathrm{~dB}$ at test frequency $2.0 \mathrm{kHz}$, but with a wide spreading. We showed that the $\mathrm{APHAB}_{\mathrm{u}}$ subscale scores were associated with the probability of hearing loss measured with audiometry. This information could enrich the expert's evaluation of the subject's hearing loss, and it might help resolve suspicious cases of aggravation. The 0.5 and $8.0 \mathrm{kHz}$ frequencies influenced hearing loss less than the frequencies in-between, and $2.0 \mathrm{kHz}$ was most influential on intermediate degree hearing loss (around $45 \mathrm{~dB}$ ), which corresponded to the frequency-dependence of speech intelligibility measured with speech audiometry.

Keywords Hearing loss - APHAB - Unaided APHAB . Hearing aid fitting · Frequency-specific hearing loss . Probability of hearing loss

\section{Introduction}

In audiology, primary diagnoses of hearing loss are based on pure tone and speech audiometry and on the results of self-reporting questionnaires. Audiometry provides a more or less objective measurement, and questionnaire scores give a subjective evaluation of impairments in hearing.

The Abbreviated Profile of Hearing Aid Benefit (APHAB), developed by Cox and Alexander in 1995, consists of 24 questions grouped into four subscales that represent everyday hearing situations [1, 2], as follows. First, the ease of communication (EC) scale represents basic hearing situations in a quiet environment without ambient noise; second, the background noise (BN) scale 
represents hearing situations with background noises; third, the reverberation (RV) scale represents hearing situations in large spaces with echoes; and fourth, the aversiveness of sounds (AV) scale measures the perception of loud events. The EC, BN, and RV subscales evaluate difficulty in understanding sounds in the situations described. In contrast, the AV subscale measures the recruitment phenomenon or hyperacusis. In 2010 and 2012, investigations on the APHAB were carried out with German-speaking patients; those studies showed no difference in the distribution from the specified US norms [3, 4]. Starting in April 2012, the APHAB became an integral part of the resources policy in Germany [5, 6]; therefore, it the most commonly used inventory in the context of diagnostics for patients with state-required insurance, which includes about $90 \%$ of the German population. Accordingly, the APHAB has the most important role under all hearing loss measuring inventories in Germany. The APHAB actually exists in 21 different languages. Differences in interpretations are not known.

The first part of the APHAB is used for primary diagnoses of hearing loss; it is designated the unaided APHAB $\left(A P H A B_{u}\right)$, because hearing problems are assessed without hearing aids. Hearing aids can be subsequently fitted, but there is no need for a second examination. That is, it is not necessary to evaluate differences in scoring between aided and unaided self-perception. Therefore, the $\mathrm{APHAB}_{\mathrm{u}}$ must be given to patients with impaired hearing at an early stage on the diagnostic pathway, along with pure tone and speech audiometry.

Recently, it was demonstrated that there was no dependency on hearing loss represented by seven standard types of audiograms and the $\mathrm{APHAB}_{\mathrm{u}}$ [7]. One the other hand, a second investigation showed that frequency-dependent hearing losses were related to some of the $\mathrm{APHAB}_{u}$ subscale scores [8]. Significant correlations between hearing loss and $\mathrm{APHAB}_{\mathrm{u}}$ scores were found at frequencies above $0.5 \mathrm{kHz}$ for the EC subscale, at all frequencies for the RV subscale, and at 1.0 and $2.0 \mathrm{kHz}$ for the AV subscale. At these frequencies, for each decibel (dB) of hearing loss, the EC and RV $\mathrm{APHAB}_{\mathrm{u}}$ scores increased by approximately 0.2 percentage points, and the AV subscale scores decreased by 0.1 percentage point. No correlation was found between the degree of hearing loss and the BN subscale score. This can be explained by the individual ability for hearing loss compensation. Hearing losses at defined single frequencies can influence the $\mathrm{APHAB}_{\mathrm{u}}$ scores. Neither of those studies demonstrated any relationship between gender, hearing loss, and the $\mathrm{APHAB}_{\mathrm{u}}$ score.

These findings due to the opposite question: is the frequency-specific hearing loss dependent on $\mathrm{APHAB}_{\mathrm{u}}$ scores and how are the belonging probabilities for each subscale?
The aim of this study is to provide a detailed investigation of correlations between the APHAB scores and the probability of having a frequency-dependent hearing loss. In addition, we investigated the probabilities of frequencydependent hearing loss (with severity grouped in steps of $5 \mathrm{~dB}$ ) at all octave frequencies, between 0.5 and $8 \mathrm{kHz}$.

\section{Methods}

An APHAB database was established in Germany several years ago [9]. At present, this database contains APHAB records and associated audiograms from thousands of individuals that were examined at more than 90 ENT clinics and practices. This database contained 7199 records of patients with impaired hearing on 23 January 2016. Records were collected, both with an online method and with paper-and-pencil records, which were later entered into the database through internet-based access. All data were stored on a central server. In all cases of subsequent hearing aid fitting, the first part of the APHAB (i.e. the $A P H A B_{u}$ ) was given to the subject before fitting the hearing aid. Thus, the APHAB was used as a primary diagnostic tool in evaluating hearing loss, as described previously [7, 8]. In addition to individual APHAB results, the database also contained the associated pure-tone audiogram data (octave frequencies between 0.5 and $8.0 \mathrm{kHz}$ ). The database did not include records for patients with a difference in hearing loss greater than $60 \mathrm{~dB}$ between the right and left ears, evaluated with the air conduction testing at frequencies at $0.5,1.0$, and $2.0 \mathrm{kHz}$, based on the three-frequency table (Table 1, [3, 4]). This exclusion avoided any influence of compensating effects, in cases of severe hearing loss asymmetry $[7,8]$. Furthermore, we eliminated records from the study, when the data were incomplete for the calculations involved in this study.

We employed a multivariate generalised linear mixed model (i.e. logistic regression with random effects [10]) to investigate the probabilities of frequency-specific hearing losses (20-75 dB hearing loss, divided into groups of 5-dB steps), within groups with different average $\mathrm{APHAB}_{\mathrm{u}}$ scores for the four subscales (EC, BN, RV, and AV). The $\mathrm{APHAB}_{\mathrm{u}}$ was administered with air conduction tests at sound frequencies of $0.5,1.0,2.0,4.0$, and $8.0 \mathrm{kHz}$. The average $\mathrm{APHAB}_{\mathrm{u}}$ scores for each subscale were sorted into groups that increased in steps of 5 percentage points. Thus, the $\mathrm{APHAB}_{\mathrm{u}}$ scores were the independent variables, and the frequency-specific audiogram results were the dependent variables. Another independent variable was gender. Calculations were performed with SAS software, version 9.4, proc glimmix. All results are presented in tables with four dimensions (or levels), as follows: first level, the average scores for each of the four $\mathrm{APHAB}_{\mathrm{u}}$ subscales 
Table 1 Three-frequency table used to define the degree of hearing impairment $[3,4]$

\begin{tabular}{|c|c|c|c|c|c|}
\hline & \multicolumn{5}{|c|}{ Hearing loss at $2.0 \mathrm{kHz}(\mathrm{dB})$} \\
\hline & $<20$ & $20-35$ & $40-55$ & $60-80$ & $>80$ \\
\hline \multicolumn{6}{|c|}{ Total hearing loss at 0.5 and $1.0 \mathrm{kHz}(\mathrm{dB})$} \\
\hline $0-35$ & None & Slight & Moderate & Moderate-profound & Profound \\
\hline $40-75$ & Slight & Slight & Moderate & Moderate-profound & Profound \\
\hline $80-115$ & Moderate & Moderate & Moderate & Moderate-profound & Profound \\
\hline $120-160$ & Moderate-profound & Moderate-profound & Moderate-profound & Moderate-profound & Profound \\
\hline$>160$ & Profound & Profound & Profound & Profound & Profound \\
\hline
\end{tabular}

Findings are from the sound audiogram of the ear with worse hearing, measured in 5-dB steps. Subjects with a difference $>60 \mathrm{~dB}$ hearing loss between the left and the right ears were initially excluded from the database

(EC, BN, RV, AV); second level, fixed combinations of the four $\mathrm{APHAB}_{\mathrm{u}}$ subscale values; third level, sound frequency (values from 0.5 to $8 \mathrm{kHz}$ ); and fourth level, the probability that hearing loss was associated with a given $\mathrm{APHAB}_{\mathrm{u}}$ subscale combination, at each sound frequency. Because the APHAB yields a vast number of possible results (each subscale contains six questions, each question is scored 1-99\%), we presented the data in tables that show the average score for each $\mathrm{APHAB}_{\mathrm{u}}$ subscale, grouped in steps of $5 \%$, for average scores of $20-80 \%$. For better understanding, we created a series of graphs showing the probabilities that a given level of hearing loss will occur at each frequency for all combinations of the $\mathrm{APHAB}_{\mathrm{u}}$ subscales (Figs. 1a-l). In addition, these figures were linked together in an animated slide show (Film 1, Online Resource 25).

Permission to store data was given voluntarily by all subjects included. The study was approved by the Ethics Committee of the Schleswig-Holstein Medical Association and the State Data Protection Officer.

\section{Results}

\section{General data}

We evaluated records of 7199 subjects, with an average age of 69.6 years [standard deviation $(\mathrm{SD}) \pm 17.0$ years]. Of these, 3081 were men $(42.8 \%$, average age $69.0 \pm 16.4$ years) and 4118 were women $(57.2 \%$, average age $70.1 \pm 17.4$ years). Some cases $(n=641)$ were not assigned to any hearing loss group, based on the threefrequency table (Table 1, [3, 4]), because the required data were incomplete for frequencies of $0.5,1.0$, and/or $2.0 \mathrm{kHz}$. These cases were not included in further assessments; thus, at least 6558 cases were available for analysis. For this study, the number of subjects that were subsequently fitted with hearing aids was irrelevant; therefore, it was not documented.
We investigated 6558 cases with a multivariate generalised linear mixed model to determine the probabilities that frequency-specific hearing losses measured at frequencies of $0.5,1.0,2.0,4.0$, and $8.0 \mathrm{kHz}$ were associated with different combinations of $\mathrm{APHAB}_{\mathrm{u}}$ values (combinations of all four APHAB subscales, $\mathrm{EC}, \mathrm{BN}, \mathrm{RV}$, and $\mathrm{AV}$, grouped in 5-percentage point steps, hearing loss severity: $20-75 \mathrm{~dB}$, analysed in 5-dB steps). We found no differences in the probabilities of hearing loss between men and women. The distribution of records among different classes of hearing loss based on the three-frequency table (Table 1) is presented in Table 2. Our analyses generated 12 tables (Tables $3 \mathrm{a}-1$ ), each with 28,561 different combinations of $\mathrm{APHAB}_{\mathrm{u}}$ subscale values. We have presented the results online (Online Resource 1-12).

\section{Associations between the probability of frequency- specific hearing loss and unaided APHAB scores}

We found that, in general, higher scores in the $\mathrm{EC}, \mathrm{BN}$, and $\mathrm{RV}$ subscales of the $\mathrm{APHAB}_{\mathrm{u}}$ were associated with higher probabilities of hearing loss (Tables 3a-1). However, increasing hearing loss severity corresponded to decreasing values of probability. The probability of hearing loss

Table 2 Distribution of hearing loss categories, based on the definitions in Table 1, among subjects in this study $(n=7199)$

\begin{tabular}{lcc}
\hline Degree of hearing loss & Percent $(\%)$ & Number \\
\hline None & 15.9 & 1146 \\
Slight & 9.5 & 682 \\
Moderate & 32.3 & 2325 \\
Moderate-profound & 27.5 & 1978 \\
Profound $_{\text {Non-attributable }}^{\mathrm{a}}$ & 5.9 & 427 \\
\hline
\end{tabular}

a 641 subjects had data that were non-attributable or incomplete, and these patients were excluded from further investigation 
associated with an $\mathrm{APHAB}_{\mathrm{u}}$ score depended on the specific $\mathrm{dB}$-value. However, the probability was lower for the lower frequencies and higher for the higher frequencies, except at $8.0 \mathrm{kHz}$, for all APHAB subscales in general.

Some interesting details emerged from this analysis. We found that the $\mathrm{APHAB}_{\mathrm{u}}$-dependent probability of a $20-\mathrm{dB}$ hearing loss was higher for 4.0 than for $8.0 \mathrm{kHz}$ sounds. Up to a 35-dB hearing loss, increasing EC values were associated with lower probabilities of a hearing loss at 4.0 and $8.0 \mathrm{kHz}$, but at a $45-\mathrm{dB}$ hearing loss that negative association was only observed for $8.0 \mathrm{kHz}$ sounds. For hearing losses above $60 \mathrm{~dB}$, the probability of a hearing loss was higher for $8.0 \mathrm{kHz}$ than for $4.0 \mathrm{kHz}$ sounds. Above $70 \mathrm{~dB}$, except at $8.0 \mathrm{kHz}$, the $\mathrm{APHAB}_{\mathrm{u}}$ scores had no detectable influence on the probability of a hearing loss. In the second step of this evaluation (Figs. 1a-1, Online Resource 13-24), we plotted the probabilities of hearing losses of 20-75 dB (in increasing steps of $5 \mathrm{~dB}$ ) against the corresponding combinations of all $\mathrm{APHAB}_{\mathrm{u}}$ subscale scores. In the third step of this evaluation, we presented the alterations in probabilities as the $\mathrm{dB}$ values increased, by connecting these figures in an animated slide show (Animation 1, Online Resource 25).

The statistical dispersion of the probabilities for hearing loss at test frequencies 4.0 and $8.0 \mathrm{kHz}$ is decreasing for a hearing loss from $20 \mathrm{~dB}$ to a value about $35-40 \mathrm{~dB}$. The probabilities for hearing loss at the test frequency $2.0 \mathrm{kHz}$ have a wide spreading for a possible middle-degree hearing loss around $45 \mathrm{~dB}$. The probability of a high-degree hearing loss is highest at test frequencies 4.0 and $8.0 \mathrm{kHz}$. But the belonging probabilities found in our study are on a very low level in these cases.

\section{Discussion}

Although investigations of the relationships between audiometric and self-reported measures began decades ago, some questions in this field remain unanswered. The association between the probability of hearing loss and questionnaire scores has only been investigated in evaluations of screening-instruments, like the HHIS-E or MAT [11, 12], to determine their specificity and sensitivity. The APHAB is a very detailed questionnaire, too large for use in screening approaches. However, the APHAB is considered highly important in the context of diagnostics for patients with state-required insurance in Germany, a population roughly comparable to patients that receive nationally funded health services in other countries.

In this study, we did not discover any significant differences between genders in how the probability of hearing loss was associated with $\mathrm{APHAB}_{\mathrm{u}}$ scores. The similarity between genders, both in distribution and average age, was consistent with findings in previous studies [7, 8].

The tables generated by this study give the probabilities that certain frequency-specific hearing losses might occur in patients that show a specific range of $\mathrm{APHAB}_{\mathrm{u}}$ values. Thus, the reader might use these original data to predict pure-tone thresholds in all octave frequencies between 0.5 and $8 \mathrm{kHz}$. In addition, Tables 3a-1 (Online resource 1-12) might allow the reader to introduce the $\mathrm{APHAB}_{\mathrm{u}}$ in diagnoses of subjects that receive statutory accident insurance. The original data could be used further to compare the results of the $\mathrm{APHAB}_{\mathrm{u}}$ scores for each subscale to the probability of the corresponding hearing loss, measured with audiometry. This information could enrich the expert's evaluation of the subject's hearing loss, and it might help resolve suspicious cases of aggravation and simulation.

Moreover, comparing $\mathrm{APHAB}_{\mathrm{u}}$ scores of the AV subscale and the probabilities of hearing loss, based on pure-tone thresholds, could be a method for measurement of subjective hyperacusis or recruitment phenomenon. High scores in the $\mathrm{AV}_{\mathrm{u}}$ should correspond to a severe hearing loss, particularly at high frequencies, and our tables can provide the associated probabilities of hearing loss. Our results have demonstrated more or less stringent associations between a possible frequencyspecific hearing loss and all $\mathrm{APHAB}_{\mathrm{u}}$ subscale scores. However, these probabilities are not equal at all frequencies, and the frequency-specific probability of hearing loss varies for different degrees of hearing loss. The probabilities we presented (Tables 3a-1, Online Resource 1-12) might enhance our understanding of the association between subjective speech intelligibility and hearing loss. Clearly, hearing losses in 0.5 and $8.0 \mathrm{kHz}$ sounds are less possible than for sounds at the frequencies in-between, and the importance of $2.0 \mathrm{kHz}$ sound was highest for a probability of an intermediate degree of hearing loss (around $45 \mathrm{~dB}$ ). These findings corresponded to the frequency-specific dependency of speech intelligibility measured with speech audiometry (reviewed in [13]).

We found that high frequencies had limited influence, and frequencies below $4.0 \mathrm{kHz}$ completely lacked influence on the probability of hearing losses of $65 \mathrm{~dB}$ and above. This finding could be explained by two components. First, only a relatively few number of subjects (5.9\%) had profound hearing loss (Table 2). Thus, in general, the probability of a severe hearing loss was low in the investigated population. Moreover, clearly, some patients reported a severe subjective hearing impairment on the $\mathrm{APHAB}_{\mathrm{u}}$, and a sharp threshold slope (dead region) was observed at high frequencies. This result pointed out the 
importance of high frequencies for speech intelligibility. In these cases, the score for the $\mathrm{EC}_{\mathrm{u}}$ subscale had little or no influence on the probability of the associated hearing loss. The widespread dispersion of the influences of 4.0 and $8.0 \mathrm{kHz}$ on a mild hearing loss and the influence of $2.0 \mathrm{kHz}$ on a moderate hearing loss could also be explained this effect.

This study provided additional information on how the $\mathrm{APHAB}_{\mathrm{u}}$ could be used by ENT surgeons and audiologists in primary measurements. In addition, the $\mathrm{APHAB}_{\mathrm{u}}$ could be used as a method for measuring the benefit of hearing aids after fitting and for diagnosing subjects that receive statutory accident insurance. Due to the wide use of the APHAB in over $90 \%$ of all severe cases of hearing loss in Germany, future studies should supplement these results with determinations of the sensitivity and specificity of the APHAB.

In a broader view, a future fourth step of evaluation could be developed. The data we provided could be compiled in a computer program or a smart phone application that could calculate the predictions of different types and degrees of hearing loss, based on the patient's answers on the APHAB questionnaire. An app like this would provide general practitioners a forecast of their patients' hearing loss and indicate the urgency of scheduling an examination by an ENT specialist and/or audiologist.

\section{Compliance with ethical standards}

Conflict of interest None for all authors.

Research involves human participants All subject participation and participation in data storage were voluntary. The research methods were approved by the Ethics Commission of the Schleswig-Holstein Medical Association and the State Data Protection Officer.

Informed consent All participants provided informed consent in writing prior to inclusion in the study. All participants signed a declaration of informed consent for storing their anonymised data.

Open Access This article is distributed under the terms of the Creative Commons Attribution 4.0 International License (http://crea tivecommons.org/licenses/by/4.0/), which permits unrestricted use, distribution, and reproduction in any medium, provided you give appropriate credit to the original author(s) and the source, provide a link to the Creative Commons license, and indicate if changes were made.

\section{References}

1. Cox RM, Alexander GC (1995) The abbreviated profile of hearing aid benefit. Ear Hear 16:176-186

2. Johnson JA, Cox RM, Alexander GC (2010) Development of APHAB norms for WDRC hearing aids and comparisons with original norms. Ear Hear 31:47-55

3. Löhler J, Frohburg R, Moser L (2010) The use of the German APHAB for quality control in hearing aid fitting in an ENToffice. Comparison of our results with the given US-norm. Laryngo Rhino Otol 89:737-744

4. Löhler J, Moser L, Heinrich D, Hörmann K, Walther LE (2012) Results of clinical use of the German version of the APHAB. HNO 60:626-636

5. Löhler J, Akcicek B, Wollenberg B, Schönweiler R (2014) Implementation of the new quality assurance agreement for the fitting of hearing aids in daily practice. Part 1: new regulations pertaining to hearing aid fitting. HNO 62:605-612

6. Löhler J, Akcicek B, Wollenberg B, Schönweiler R (2014) Implementation of the new quality assurance agreement for the fitting of hearing aids in daily practice. Part 2: new diagnostic aspects of speech audiometry. HNO 62:667-682

7. Löhler J, Akcicek B, Wollenberg B, Kappe T, Schlattmann P, Schlattmann P, Schönweiler R (2016) The influence of hearing loss represented by standard audiogram types to unaided APHAB scores. Laryngo Rhino Otol 95:540-545

8. Löhler J, Akcicek B, Wollenberg B, Kappe T, Schönweiler R (2016) The influence of frequency-dependent hearing loss to unaided APHAB scores. Eur Arch Oto Rhin Laryngol. doi:10. 1007/s00405-016-3966-9

9. Löhler J, Akcicek B, Kappe T, Schlattmann P, Wollenberg B, Schönweiler R (2014) Development and use of an APHAB database. HNO 62:735-745

10. Fahrmeir L, Tutz G (2001) Multivariate statistical modelling based on generalised linear models. Springer, Heidelberg

11. Ventry IM, Weinstein BE (1982) The hearing handicap inventory for the elderly: a new tool. Ear Hear 3:128-134

12. Löhler J, Schlattmann P, Walther LE (2013) The Mini-AudioTest (MAT). Screening inventory for hearing loss starting at the age of 50. Oto Rhino Laryngol 92:815-822

13. Hornsby BWY, Ricketss TA (2006) The effects of hearing loss on the contribution of high- and low-frequency speech information to speech understanding. II. Sloping hearing loss. J Acoust Soc Am 119:1752-1763 